\title{
Increased oxidative stress is associated with insulin resistance and infertility in polycystic ovary syndrome
}

\author{
Alev Özer, Murat Bakacak, Hakan Kıran, Önder Ercan, Bülent Köstü, \\ Mine Kanat-Pektaş, Metin Kılınç, Ferhat Aslan \\ Sütçü Imam University, Turkey
}

\begin{abstract}
Objectives: The present study aims to investigate the role of oxidant-antioxidant status in young women with polycystic ovary syndrome (PCOS).

Material and methods: Seventy-one women with PCOS and 53 healthy controls are compared in aspect of demographic characteristics, biochemical data, hormones, and oxidant-antioxidant status.

Results: The PCOS group had significantly lower zinc, higher malondialdehyde and gluthathione peroxidase and lower serum catalase levels than the control group $(p=0.016, p<0.001, p=0.043$ and $p=0.025$ respectively). The PCOS patients with IR had significantly higher malondialdehyde, lower catalase and serum zinc levels than the PCOS patients without IR $(p=0.015, p=0.010, p=0.001$ respectively). The infertile PCOS patients had significantly higher malondialdehyde, lower catalase and serum zinc levels than the fertile PCOS patients $(p=0.022, p=0.045, p=0.001$ respectively). There was a statistically significant and positive correlation between HOMA-IR and malondialdehyde values $(r=0.523, p=0.001)$, between HOMA-IR and glutathione peroxidase values $(r=0.468, p=0.001)$ and between HOMA-IR and zinc values $(r=0.601, p=0.001)$. There was a statistically significant and negative correlation between HOMA-IR and catalase values $(r=-0.493, p=0.001)$. Conclusions: The patients with PCOS are under oxidative stress and this oxidative stress seems to be the highest in patients with IR and with infertility. Despite the prominent increase in the oxidative stress, there was a variation in the antioxidant response.
\end{abstract}

Key words: infertility, insulin resistance, oxidative stress, polycystic ovary syndrome

Ginekologia Polska 2016; 87, 11: 733-738

\section{INTRODUCTION}

Polycystic ovary syndrome is the most common endocrinopathy in women of reproductive age with a prevalence of $5-10 \%$. This syndrome most frequently occurs as the interactions among the central nervous system, pituitary gland, adrenal glands, ovaries and extra-glandular tissues are impaired. Having a chronic course, PCOS has a negative impact on the quality of life over years. Moreover, PCOS may occur concurrently with hyperandrogenism, hyperinsulinemia, and glucose intolerance, leading to conditions such as infertility, recurrent spontaneous abortions, hyperlipidemia, type 2 diabetes mellitus, hypertension, coronary atherosclerosis, endometrial hyperplasia, and endometrial cancer [1-4].

It has been hypothesized that oxidative stress participates in the pathogenesis of endometriosis, unexplained infertility, male factor infertility, anovulation, and the impairment of oocyte quality in the human reproductive system [5-8]. Therefore, the question has risen about the role of oxidative stress in the pathogenesis of PCOS and related complications [9]. Oxidative stress is defined as the disturbance in the balance between antioxidant defense mechanisms and reactive oxygen species (ROS). This imbalance leads to an increase in ROS, which damages the cell membrane lipids, leading to lipid peroxidation by the formation of malondialdehyde (MDA). It has been demonstrated that oxidative stress is generally enhanced in the tissues of the animals with zinc deficiency and copper abundance [10].

The harmful effects of an increased oxidative load are reduced by antioxidant enzymes such as superoxide dismutase (SOD) and catalase (CAT) that convert ROS to less 
harmful molecules. When the production of excessive ROS overcomes the natural antioxidant defense system, an unstable environment may emerge for the reproductive cells and tissues $[11,12]$. Therefore; it is important to assess the oxidant/antioxidant status in PCOS patients.

\section{OBJECTIVE}

The present study aims to investigate the role of oxidant-antioxidant status in young women who are diagnosed with PCOS

\section{MATERIAL AND METHODS}

This descriptive study was approved by the Ethical Committee and Institutional Review Board of Kahramanmaras Sutcu Imam University where it was conducted between January 2014 and December 2015. Written informed consent was obtained from each participant.

Patient Selection and Study Design

This study compares the demographic characteristics, biochemical data, hormones and oxidant-antioxidant status of 71 women with PCOS and 53 healthy controls. The inclusion criteria included the age between 20 and 30 years, the absence of major medical disorders (endocrinologic or neoplastic), and the presence of clinical and biochemical findings of hyperandrogenism in the absence of the ovarian, adrenal and hypophyseal pathologies.

The diagnosis of PCOS was based on the presence of two of the three following criteria: (1) the presence of oligomenorrhea or amenorrhea, (2) clinical and/or biochemical hyperandrogenism, (3) polycystic ovaries on ultrasonography [13].

Healthy controls had regular menstrual cycles lasting 25-35 days, normal serum androgen concentrations and mid-luteal serum progesterone level consistent with ovulation ( $>5 \mathrm{ng} / \mathrm{mL}$ ). The women who smoked, the women who had body mass indices $>30 \mathrm{~kg} / \mathrm{m}^{2}$, and the women who were using contraceptive methods or any medication for ovulation were excluded.

Subgroup analysis was done according to the insulin resistance which was calculated using the HOMA-IR index (fasting glucose $x$ fasting insulin/constant). Since fasting glucose value was measured in $\mathrm{mg} / \mathrm{dL}$, the constant was set at 405. HOMA-IR $>2.5$ was accepted as insulin resistance There were 33 PCOS patients with insulin resistance and 38 PCOS patients without insulin resistance. Subgroup analysis was also done based on the fertility status. There were 41 PCOS patients who did not become pregnant despite one year of unprotected sexual intercourse and 30 PCOS patients who became spontaneously pregnant.

\section{Laboratory studies}

Venous blood samples were obtained from the antecubital area by phlebotomy between the hours of 08:00 and
09:00 following 10-12 h of fasting. After these samples were allowed to clot, they were centrifuged for 10 minutes at $3000 \mathrm{~g}$. The sera were separated and stored at $-70^{\circ} \mathrm{C}$ until the analysis.

Plasma glucose levels were measured by enzymatic colorimetric assay (GOD-PAP, Roche Diagnostics, Mannheim). Plasma zinc and copper levels were measured by atomic absorption spectrophotometry (ContrAA700, Analytik Jena AG, Germany) with an intra-assay CV of $2.4 \%$ and an inter-assay $\mathrm{CV}$ of $3.1 \%$. Electrochemoluminescence immunoassay was used to measure serum levels of follicle stimulating hormone $(\mathrm{FSH})$, luteinizing hormone (LH), estradiol, prolactin and 17-hydroxy-progesterone (Roche Elecsys 1010/2010, Roche Diagnostics, Mannheim, Germany). The serum concentrations of dehidroepiandrosterone sulphate (DHEAS), thyroid stimulating hormone (TSH), total testosterone, free testosterone and insulin were measured by radioimmunoassay (DSL Diagnostic Systems Laboratories, USA). The intra-assay CVs were $5.3 \%, 3.8 \%, 7.8 \%, 6.2 \%, 5.0 \%, 9.6 \%, 10.0 \%, 9.6 \%, 9.7 \%$ and $8.2 \%$ whereas the inter-assay CVs were $1.8 \%, 1.5 \%, 10.0 \%$, $5.7 \%, 4.1 \%, 9.3 \%, 7.8 \%, 6.6 \%, 6.2 \%$ and $7.4 \%$ for $\mathrm{FSH}, \mathrm{LH}$, estradiol, prolactin, 17-hydroxy-progesterone, DHEAS, TSH, total testosterone, free testosterone and insulin respectively.

\section{Oxidant-antioxidant status}

After blood samples were centrifuged at $4^{\circ} \mathrm{C}$ at $3,000 \mathrm{rpm}$ for 10 minutes, plasma samples were separated as aliquots and stored at $-80^{\circ} \mathrm{C}$ until the analysis. After removal of plasma, the packed erythrocytes were washed 3 times with $9 \mathrm{~g} / \mathrm{L} \mathrm{NaCl}$ solution and hemolyzed with ice-cold distilled water $(1 / 5, \mathrm{v} / \mathrm{v})$.

The malondialdehyde measurements were performed using the method described by Yagi [14]. This method is based on the spectrophotometric measurement of the red color in the thiobarbituric acid-malondialdehyde complex at $532 \mathrm{~nm}$ after the erythrocyte lysate is deproteinized with trichloroacetic acid. The results were calculated as $\mathrm{nmol} / \mathrm{mL}$.

After the erythrocyte lysate was diluted to 1:100 with phosphate buffer, the catalase activity was measured with a Shimadzu UV-1208 spectrophotometer (Kyoto, Japan) using the method described by Aebi [15]. This method is based on the decomposition of hydrogen peroxide by catalase. The results were computed as $\mu \mathrm{g} / \mathrm{mL}$.

Superoxide dismutase was measured using a Shimadzu UV-1208 spectrophotometer using the method described by Sun et al. [16]. This method is based on the inhibition of nitroblue tetrazolium reduction with xanthine-xanthine oxidase which is utilized as a superoxide generator. The specific activity was calculated as $\mu \mathrm{g} / \mathrm{mL}$.

Glutathione peroxidase was measured in tissue samples using a Shimadzu UV-1208 spectrophotometer using the method described by Awasthi et al. [17]. This method is 
Table 1. Demographic, biochemical and oxidant-antioxidant status of the participants

\begin{tabular}{|c|c|c|c|}
\hline & $\begin{array}{l}\text { Polycystic ovary syndrome } \\
\qquad(\mathrm{n}=71)\end{array}$ & $\begin{array}{l}\text { Healthy controls } \\
\qquad(n=53)\end{array}$ & P value \\
\hline Age (years) & $26.2 \pm 5.5$ & $29.4 \pm 8.8$ & 0.097 \\
\hline Body mass index $\left[\mathrm{kg} / \mathrm{m}^{2}\right]$ & $26.9 \pm 5.4$ & $23.8 \pm 4.7$ & 0.059 \\
\hline Plasma glucose [mg/dL] & $90.2 \pm 6.1$ & $78.5 \pm 7.1$ & $0.004^{*}$ \\
\hline Insulin [mIU/L] & $13.18 \pm 8.9$ & $9.7 \pm 6.4$ & $0.015^{*}$ \\
\hline HOMA-IR & $2.92 \pm 2.15$ & $1.88 \pm 1.68$ & $0.040^{*}$ \\
\hline Follicle stimulating hormone [mIU/mL] & $5.84 \pm 2.11$ & $6.38 \pm 2.45$ & 0.250 \\
\hline Luteinizing hormone [mIU/mL] & $7.95 \pm 5.06$ & $4.48 \pm 2.03$ & $0.001^{*}$ \\
\hline Estradiol [pg/mL] & $48.01 \pm 5.23$ & $48.0 \pm 4.85$ & 0.990 \\
\hline Prolactin [ng/mL] & $23.39 \pm 2.15$ & $18.06 \pm 1.34$ & 0.150 \\
\hline DHEAS $[\mu \mathrm{g} / \mathrm{dL}]$ & $341.2 \pm 87.4$ & $366.7 \pm 71.9$ & 0.130 \\
\hline 17-hydroxy-progesterone [ng/mL] & $0.8 \pm 0.5$ & $0.6 \pm 0.2$ & 0.632 \\
\hline Total testosterone $[\mathrm{ng} / \mathrm{mL}]$ & $1.55 \pm 0.91$ & $0.89 \pm 0.34$ & $0.001^{*}$ \\
\hline Free testosterone $[\mathrm{ng} / \mathrm{mL}]$ & $2.31 \pm 0.54$ & $1.46 \pm 0.22$ & $0.001^{*}$ \\
\hline Thyroid stimulating hormone $[\mu \mathrm{lU} / \mathrm{mL}]$ & $2.02 \pm 1.36$ & $3.31 \pm 1.84$ & 0.700 \\
\hline Malondialdehyde [nmol/mL] & $23.0 \pm 13.8$ & $3.1 \pm 2.7$ & $<0.001^{*}$ \\
\hline Catalase $[\mu \mathrm{g} / \mathrm{mL}]$ & $1875.7 \pm 435.1$ & $2302.8 \pm 437.3$ & $0.025^{*}$ \\
\hline Superoxide dismutase $[\mu \mathrm{g} / \mathrm{mL}]$ & $31.0 \pm 9.0$ & $31.1 \pm 8.6$ & 0.988 \\
\hline Gluthathione peroxidase $[\mu \mathrm{g} / \mathrm{mL}]$ & $3.6 \pm 0.4$ & $3.2 \pm 0.5$ & $0.043^{*}$ \\
\hline Serum zinc $[\mu \mathrm{g} / \mathrm{dL}]$ & $84.4 \pm 25.5$ & $99.4 \pm 19.9$ & $0.016^{*}$ \\
\hline Serum copper $[\mu \mathrm{g} / \mathrm{dL}]$ & $85.8 \pm 29.5$ & $80.3 \pm 32.5$ & 0.465 \\
\hline
\end{tabular}

${ }^{*} p<0.05$ was accepted to be statistically significant

based on the decrease in the absorbance at $340 \mathrm{~nm}$. The results were computed as $\mu \mathrm{g} / \mathrm{mL}$.

\section{Statistical analysis}

Collected data were analyzed by the Statistical Package for Social Sciences version 18.0 (SPSS Inc., Chicago, IL, USA). Continuous variables were expressed as mean \pm standard deviation (range: minimum-maximum) and categorical variables were denoted as numbers or percentages where appropriate. Smirnov-Kolmogorov test was used to test the distribution of the variables. Student t-test, one-way analysis of variance, Mann Whitney $\mathrm{U}$ test and Kruskal-Wallis test were used for the comparisons. A post hoc analysis was carried out to determine the two variables between which there is a statistically significant difference. Two-tailed $p$ values less than 0.05 were accepted to be statistically significant.

\section{RESULTS}

This study compares the demographic and clinical characteristics of 71 women with PCOS and 53 healthy controls. When compared with the healthy controls, the women with PCOS had significantly lower serum zinc levels and catalase activity ( $p=0.016$ and $p=0.025$ respectively) as well as significantly higher malondialdehyde and gluthathione peroxidase levels ( $<0.001$ and $p=0.043$ respectively) (Table 1).

There were 33 PCOS patients with insulin resistance and 38 PCOS patients without insulin resistance. When compared with the healthy controls, PCOS patients with insulin resistance had significantly lower catalase activity $(p=0.008)$ and significantly lower serum zinc, malondialdehyde and gluthathione peroxidase levels $(p=0.001, p<0.001$ and $p=0.024$ respectively)

When compared to PCOS patients without insulin resistance, the PCOS patients with insulin resistance had significantly higher malondialdehyde levels, significantly lower catalase activity and serum zinc levels $(p=0.15, p=0.10$, and $p=0.001$ respectively) (Table 2 ).

There were 41 PCOS patients with infertility and 30 PCOS patients without infertility. When compared with the healthy controls, the infertile PCOS patients had significantly higher lower concentrations of serum zinc levels and catalase activity ( $p=0.001, p=0.050$ respectively) as well as significantly higher malondialdehyde and gluthathione peroxidase levels ( $p<0.001$ and $p=0.004$ respectively). When compared to fertile PCOS patients, infertile PCOS patients had signifi- 
Table 2. Characteristics of the participants with respect to insulin resistance

\begin{tabular}{|c|c|c|c|c|}
\hline & $\begin{array}{c}\text { IR(+) PCOS } \\
(n=33)\end{array}$ & $\begin{array}{c}\text { IR(-) PCOS } \\
(n=38)\end{array}$ & $\begin{array}{l}\text { Healthy controls } \\
\qquad(n=53)\end{array}$ & $P$ value \\
\hline Age (years) & $26.3 \pm 5.6$ & $26.1 \pm 5.4$ & $29.4 \pm 8.8$ & 0.077 \\
\hline Body mass index $\left[\mathrm{kg} / \mathrm{m}^{2}\right]$ & $27.7 \pm 5.6$ & $25.2 \pm 5.2$ & $23.8 \pm 4.7$ & $0.049 *$ \\
\hline Plasma glucose [mg/dL] & $96.5 \pm 6.9$ & $88.1 \pm 5.7$ & $78.5 \pm 7.1$ & $0.027^{*}, 0.050^{\dagger}$ \\
\hline Insulin [mIU/L] & $16.8 \pm 9.0$ & $10.1 \pm 8.6$ & $9.7 \pm 6.4$ & $0.001^{* \dagger}$ \\
\hline HOMA-IR & $4.01 \pm 2.83$ & $2.27 \pm 2.14$ & $1.88 \pm 1.68$ & $<0.001^{*}, 0.002^{\dagger}$ \\
\hline $\mathrm{FSH}[\mathrm{mlU} / \mathrm{mL}]$ & $5.78 \pm 2.19$ & $5.90 \pm 2.04$ & $6.38 \pm 2.45$ & 0.255 \\
\hline $\mathrm{LH}[\mathrm{mlU} / \mathrm{mL}]$ & $8.15 \pm 4.92$ & $7.75 \pm 5.12$ & $4.48 \pm 2.03$ & $0.001^{*}$ \\
\hline Estradiol $[\mathrm{pg} / \mathrm{mL}]$ & $50.05 \pm 5.13$ & $46.02 \pm 4.78$ & $48.0 \pm 4.85$ & 0.990 \\
\hline Prolactin [ng/mL] & $25.39 \pm 2.15$ & $21.24 \pm 2.54$ & $18.06 \pm 1.34$ & 0.150 \\
\hline DHEAS $[\mu \mathrm{g} / \mathrm{dL}]$ & $351.2 \pm 91.4$ & $318.9 \pm 89.0$ & $366.7 \pm 71.9$ & 0.130 \\
\hline 17-OH-P [ng/mL] & $1.1 \pm 0.6$ & $0.7 \pm 0.4$ & $0.6 \pm 0.2$ & 0.514 \\
\hline Total testosterone $[\mathrm{ng} / \mathrm{mL}]$ & $1.79 \pm 1.15$ & $1.33 \pm 0.86$ & $0.85 \pm 0.34$ & $0.030^{*}$ \\
\hline Free testosterone $[\mathrm{ng} / \mathrm{mL}]$ & $2.51 \pm 0.74$ & $2.08 \pm 0.53$ & $1.46 \pm 0.22$ & $0.040^{*}$ \\
\hline $\mathrm{TSH}[\mu \mathrm{IU} / \mathrm{mL}]$ & $2.14 \pm 1.36$ & $2.00 \pm 1.11$ & $3.31 \pm 1.84$ & 0.766 \\
\hline Malondialdehyde [nmol/mL] & $31.1 \pm 20.7$ & $14.4 \pm 6.3$ & $3.1 \pm 2.7$ & $<0.001^{*}, 0.015^{\dagger}$ \\
\hline Catalase $[\mu \mathrm{g} / \mathrm{mL}]$ & $1278.9 \pm 235.1$ & $2294.3 \pm 652.4$ & $2302.8 \pm 437.9$ & $0.008^{*}, 0.010^{\dagger}$ \\
\hline Superoxide dismutase $[\mu \mathrm{g} / \mathrm{mL}]$ & $31.9 \pm 9.5$ & $30.5 \pm 8.2$ & $31.1 \pm 8.6$ & 0.988 \\
\hline $\mathrm{GTP}[\mu \mathrm{g} / \mathrm{mL}]$ & $3.9 \pm 0.7$ & $3.6 \pm 0.4$ & $3.2 \pm 0.5$ & $0.024^{*}$ \\
\hline Serum zinc $[\mu \mathrm{g} / \mathrm{dL}]$ & $70.3 \pm 24.7$ & $98.1 \pm 31.5$ & $99.4 \pm 19.9$ & $0.001^{* \dagger}$ \\
\hline Serum copper $[\mu \mathrm{g} / \mathrm{dL}]$ & $89.0 \pm 28.4$ & $82.7 \pm 30.3$ & $80.3 \pm 32.5$ & 0.169 \\
\hline
\end{tabular}

cantly higher malondialdehyde levels, lower catalase activity and serum zinc levels $(p=0.022, p=0.045, p=0.001$ for each) (Table 3).

There was a statistically significant and positive correlation between HOMA-IR and malondialdehyde values $(r=0.523, p=0.001)$, between HOMA-IR and glutathione peroxidase values $(r=0.468, p=0.001)$ and between HOMA-IR and zinc values $(r=601, p=0.001)$. There was a statistically significant and negative correlation between HOMA-IR and catalase values $(r=-0.493, p=0.001)$.

\section{DISCUSSION}

Oxidative stress may cause changes in biological molecules and these changes may have cumulative effects on biological structures which may refer to a hazardous impact on cells, tissues and organs. Oxidative stress is associated with the pathogenesis of several chronic diseases including diabetes mellitus, atherosclerosis and ischemia-reperfusion injury [6-9].

Recently published studies have focused on the effects of oxidative stress on the female reproductive system. It has been reported that superoxide dismutase participates in the implantation process and the presence of antioxidants in follicular fluid directly correlates with the success of in vitro fertilization $[18,19]$. Therefore, it has been suggested that adoption of a healthy diet, utilization of multivitamins, and avoidance from alcohol, smoking and caffeine may help to provide a balanced oxidative-antioxidative environment and, thus, to enhance fertility in the female reproductive system [20].

Oxidative stress is increased in PCOS patients. In an Iran study, the women with PCOS were found to have significantly higher advanced oxidation protein products and significantly lower total antioxidant status when compared to those of the age- and body mass index-matched healthy controls [9].

Oxidative stress in PCOS patients was previously linked to obesity [21,22]. However, a few other studies were able to detect increased oxidative stress even in lean PCOS patients $[23,24]$. That's why; insulin resistance has been implicated as a causal factor the increased oxidative stress in affected women. Insulin resistance causes hyperglycemia which triggers the release of reactive oxygen species from the mononuclear cells and, thus, induces oxidative stress. Oxidative stress leads to cellular damage and activates the transcription of pro-inflammatory cytokines such as tumor necrosis 
Table 3. Characteristics of the participants with respect to fertility

\begin{tabular}{|c|c|c|c|c|}
\hline & $\begin{array}{l}\text { Infertile PCOS } \\
\quad(n=41)\end{array}$ & $\begin{array}{l}\text { Fertile PCOS } \\
\qquad(n=30)\end{array}$ & $\begin{array}{l}\text { Healthy controls } \\
\qquad(n=53)\end{array}$ & $P$ value \\
\hline Age (years) & $26.3 \pm 5.6$ & $26.1 \pm 5.4$ & $29.4 \pm 8.8$ & 0.077 \\
\hline Body mass index $\left[\mathrm{kg} / \mathrm{m}^{2}\right]$ & $28.4 \pm 5.9$ & $26.3 \pm 4.8$ & $23.8 \pm 4.7$ & $0.005^{*}$ \\
\hline Plasma glucose [mg/dL] & $91.5 \pm 6.9$ & $87.1 \pm 5.7$ & $78.5 \pm 7.1$ & $0.034^{*}, 0.049^{\dagger}$ \\
\hline Insulin [mIU/L] & $18.4 \pm 8.9$ & $13.1 \pm 8.2$ & $9.7 \pm 6.4$ & $0.021^{*}, 0.031^{\dagger}$ \\
\hline HOMA-IR & $4.16 \pm 2.83$ & $2.79 \pm 2.12$ & $1.88 \pm 1.68$ & $<0.001^{*}, 0.027^{\dagger}$ \\
\hline $\mathrm{FSH}[\mathrm{mIU} / \mathrm{mL}]$ & $6.14 \pm 2.19$ & $5.54 \pm 2.04$ & $6.38 \pm 2.45$ & 0.238 \\
\hline $\mathrm{LH}[\mathrm{mlU} / \mathrm{mL}]$ & $7.93 \pm 4.92$ & $7.45 \pm 5.12$ & $4.48 \pm 2.03$ & $0.001^{*}$ \\
\hline Estradiol [pg/mL] & $49.95 \pm 5.21$ & $47.12 \pm 4.52$ & $48.0 \pm 4.85$ & 0.988 \\
\hline Prolactin [ng/mL] & $23.39 \pm 2.33$ & $24.24 \pm 2.66$ & $18.06 \pm 1.34$ & 0.176 \\
\hline DHEAS $[\mu \mathrm{g} / \mathrm{dL}]$ & $388.2 \pm 87.4$ & $338.6 \pm 90.2$ & $366.7 \pm 71.9$ & 0.144 \\
\hline 17-OH-P [ng/mL] & $1.0 \pm 0.6$ & $0.9 \pm 0.4$ & $0.6 \pm 0.2$ & 0.073 \\
\hline Total testosterone $[\mathrm{ng} / \mathrm{mL}]$ & $1.63 \pm 1.12$ & $1.44 \pm 0.87$ & $0.85 \pm 0.34$ & $0.042^{*}$ \\
\hline Free testosterone $[\mathrm{ng} / \mathrm{mL}]$ & $2.91 \pm 0.94$ & $2.38 \pm 0.44$ & $1.46 \pm 0.22$ & $0.044^{*}$ \\
\hline $\mathrm{TSH}[\mu \mathrm{IU} / \mathrm{mL}]$ & $2.10 \pm 1.26$ & $1.99 \pm 1.64$ & $3.31 \pm 1.84$ & 0.850 \\
\hline Malondialdehyde [nmol/mL] & $32.1 \pm 20.7$ & $10.4 \pm 6.3$ & $3.1 \pm 2.7$ & $<0.001^{*}, 0.022^{\dagger}$ \\
\hline Catalase $[\mu \mathrm{g} / \mathrm{mL}]$ & $1488.7 \pm 253.1$ & $2471.3 \pm 670.1$ & $2302.8 \pm 437.9$ & $0.050^{*}, 0.045^{\dagger}$ \\
\hline Superoxide dismutase $[\mu \mathrm{g} / \mathrm{mL}]$ & $32.0 \pm 9.7$ & $29.3 \pm 10.2$ & $31.1 \pm 8.6$ & 0.880 \\
\hline $\mathrm{GTP}[\mu \mathrm{g} / \mathrm{mL}]$ & $4.0 \pm 1.1$ & $3.4 \pm 0.2$ & $3.2 \pm 0.5$ & $0.004^{*}$ \\
\hline Serum zinc $[\mu \mathrm{g} / \mathrm{dL}]$ & $68.7 \pm 26.4$ & $100.2 \pm 33.3$ & $99.4 \pm 19.9$ & $0.001^{\dagger *}$ \\
\hline Serum copper $[\mu \mathrm{g} / \mathrm{dL}]$ & $88.2 \pm 29.2$ & $83.9 \pm 31.3$ & $80.3 \pm 32.5$ & 0.477 \\
\hline $\begin{array}{l}\text { *There is statistically significant diffe } \\
\text { 'There is statistically significant diffe } \\
\text { IR - insulin resistance, PCOS - poly } \\
\text { sulphate, } 17-\mathrm{OH}-\mathrm{P}-17 \text {-hydroxy-pro }\end{array}$ & $\begin{array}{l}\mathrm{R}(+) \mathrm{PCOS} \text { and hea } \\
\mathrm{R}(+) \text { and IR (-) PCO } \\
\text { me, FSH-follicle st } \\
\text { hyroid stimulating }\end{array}$ & $\begin{array}{l}\text { groups } \\
\text { hormone, LH - lu } \\
\text { GTP - gluthathio }\end{array}$ & $\begin{array}{l}\text { ormone, DHEAS - } \\
\text { se }\end{array}$ & androsterone \\
\hline
\end{tabular}

factor-alpha which is a known mediator of insulin resistance. This pro-inflammatory state may contribute to the development of insulin resistance and hyperandrogenism as well [25].

In a Turkish study, the PCOS patients with insulin resistance were found to have significantly higher malondialdehyde levels, significantly lower thiol levels, significantly lower superoxide dismutase activity and lower catalase activity than the PCOS patients without insulin resistance [26]. The present study also reports that PCOS patients with insulin resistance had significantly higher malondialdehyde levels, significantly lower catalase activity and serum zinc levels when compared to the PCOS patients without insulin resistance.

The significantly higher malondialdehyde levels and significantly lower zinc levels may indicate increased oxidative stress in relation with insulin resistance for the PCOS patients. On the contrary, lower catalase levels may indicate the inhibition of antioxidant enzymes in PCOS patients with insulin resistance. Such an inhibition may be related with the existence of advanced stage PCOS which might have prevented any alterations to take place in the antioxidant enzyme concentrations.

It is well known that zinc plays a critical role in the function of metalloproteins by activating oxidoreductases, hy- drolases, ligases and lyases. This element also participates in insulin synthesis secretion, signaling and metabolism and acts together with copper in the functions of superoxide dismutase and catalase. Therefore, zinc deficiency may have a role as either an initiator or promoter of the underlying mechanisms and metabolic features of PCOS by means of causing insulin resistance, decreasing antioxidant capacity and inducing apoptosis. Moreover, it is possible that decreased antioxidant capacity can be aggravated by zinc deficiency and insulin resistance in women with PCOS [27].

Turan et al. were the first to investigate the relationship between oxidative stress markers and fertility status in PCOS patients. They found that the malondialdehyde levels were significantly higher and thiol levels were significantly lower in infertile PCOS patients when compared to fertile PCOS patients. Accordingly, it has been concluded that lower thiol levels and higher antioxidant enzyme levels might point out a compensatory antioxidative mechanism in the reviewed young and lean patients [26].

As for the present study, the PCOS patients with infertility had significantly higher malondialdehyde levels, significantly lower catalase levels and serum zinc concentrations than those of the fertile PCOS patients. These significantly 
higher malondialdehyde levels and significantly lower zinc levels may reflect increased oxidative stress and impaired ovulation within the ovaries of PCOS patients. However, lower catalase levels may show the determent of any antioxidant enzymes in women with PCOS.

The studies aiming to assess the oxidant-antioxidant status in PCOS patients demonstrated disruption in the oxidant-antioxidant balance in these patients. Moreover, there was a variation in the antioxidant response despite the prominent increase in the oxidative stress. This variation could be attributed to the differences in the demographic and genetic differences in the expression of antioxidant enzymes [24].

\section{CONCLUSIONS}

In conclusion, the patients with PCOS are under oxidative stress and this oxidative stress seems to be the highest in patients with insulin resistance and patients with infertility. The power of this conclusion is limited by the relatively small sample size, the concurrent existence of insulin resistance and infertility in a relatively higher proportion of the PCOS patients. Further research is warranted to clarify the role of the oxidative stress in the pathogenesis of PCOS.

\section{REFERENCES}

1. Mortada R, Williams T. Metabolic syndrome: Polycystic ovary syndrome. FP Essent. 2015, 435, 30-42.

2. Dumitrescu R, Mehedintu C, Briceag I, [et al.]. The polycystic ovary syndrome: an update on metabolic and hormonal mechanisms. J Med Life. 2015, 8, 142-145.

3. Taghavi SA, Bazarganipour F, Montazeri A, [et al.]. Health-related quality of life in polycystic ovary syndrome patients: A systematic review. Iran J Reprod Med. 2015, 13, 473-482.

4. Carmina E. Reproductive system outcome among patients with polycystic ovarian syndrome. Endocrinol Metab Clin North Am. 2015, 44, 787-797.

5. Sharma I, Dhaliwal LK, Saha SC, [et al.]. Role of 8-iso-prostaglandin F2-alpha and 25-hydroxycholesterol in the pathophysiology of endometriosis. Fertil Steril. 2010, 94, 63-70.

6. Hosen MB, Islam MR, Begum F, [et al.]. Oxidative stress induced sperm DNA damage, a possible reason for male infertility. Iran J Reprod Med. 2015,13, 525-532.

7. Kazemi A, Ramezanzadeh F, Nasr Esfahani, [et al.]. Relationship between energy expenditure related factors and oxidative stress in follicular fluid. Int J Fertil Steril. 2014, 8, 175-182.
8. Dumesic DA, Meldrum DR, Katz-Jaffe MG, [et al.]. Oocyte environment: follicular fluid and cumulus cells are critical for oocyte health. Fertil Steril. 2015, 103, 303-316.

9. Moti M, Amini L, Mirhoseini Ardakani SS, [et al.]. Oxidative stress and anti-oxidant defense system in Iranian women with polycystic ovary syndrome. Iran J Reprod Med. 2015, 13, 373-378.

10. Djuric A, Begic A, Gobeljic B, [et al.]. Oxidative stress, bioelements and androgen status in testes of rats subacutely exposed to cadmium. Food Chem Toxicol. 2015, 86, 25-33.

11. Fujii J, luchi Y, Okada F. Fundamental roles of reactive oxygen species and protective mechanisms in the female reproductive system. Reprod Biol Endocrinol. 2005, 2, 43.

12. Ayaz A, Agarwal A, Sharma R, [et al.]. Impact of precise modulation of reactive oxygen species levels on spermatozoa proteins in infertile men. Clin Proteomics. 2015, 12, 4.

13. Rotterdam ESHRE/ASRM-Sponsored PCOS Consensus Workshop Group Revised 2003 consensus on diagnostic criteria and long-term health risks related to polycystic ovary syndrome. Fertil Steril. 2004, 81, 19-25.

14. YagiK.Asimplefluorometricassay forlipoperoxidein blood plasma.Biochem Res. 1976, 15, 212-216.

15. Aebi H. Catalase. In Methods of Enzymatic Analysis. Bergmeyer H-U (ed). 1974, 673-678.

16. Sun Y, Oberley LW, Li Y. A simple method for clinical assay of superoxide dismutase. Clin Chem. 1988, 34, 497-500.

17. Awasthi YC, Beutler E, Srivastava SK. Purification and properties of human erythrocyte glutathione peroxidase.J Biol Chem. 1975, 250, 5144-5149.

18. Sugino N, Karube-Harada A, Sakata A, [et al.]. Different mechanisms for the induction of copper-zinc superoxide dismutase and manganesesuperoxide dismutase by progesterone in human endometrial stromal cells. Hum Reprod. 2002, 17, 1709-1714.

19. Ruder EH, Hartman TJ, Blumberg J, [et al.]. Oxidative stress and antioxidants: exposure and impact on female fertility. Hum Reprod Update. 2008, 14, 345-357.

20. Ruder EH, Hartman TJ, Goldman MB. Impact of oxidative stress on female fertility. Curr Opin Obstet Gynecol. 2009, 21, 219-222.

21. Fenkci V, Fenkci S, Yilmaz M, [et al.]. Decreased total antioxidant status and increased oxidative stress in women with polycystic ovary syndrome may contribute to the risk of cardiovascular disease. Fertil Steril. 2003, 80, 123-127.

22. Liu J, Zhang. The role of oxidative stress in the pathogenesis of polycystic ovary syndrome. Sichuan Da Xue Xue Bao Yi Xue Ban. 2012, 43, 187-190.

23. Yilmaz M, Bukan N, Ayvaz G, [et al.]. The effects of rosiglitazone and metformin on oxidative stress and homocysteine levels in lean patients with polycystic ovary syndrome. Hum Reprod. 2005, 20, 3333-3340.

24. Kuscu NK, Var A. Oxidative stress but not endothelial dysfunction exists in nonobese, young group of patients with polycystic ovary syndrome. Acta Obstet. Gynecol Scand. 2009, 88, 612-617.

25. Desai V, Prasad NR, Manohar SM, [et al.]. Oxidative stress in non-obese women with polycystic ovarian syndrome. J Clin Diagn Res. 2014, 8, CC01-3.

26. Turan V, Sezer ED, Zeybek B, [et al.]. Infertility and the presence of insulin resistance are associated with increased oxidative stress in young, non-obese Turkish women with polycystic ovary syndrome. J Pediatr Adolesc Gynecol. 2015, 28, 119-123.

27. Zheng G, Wang L, Guo Z, [et al.]. Associations of serum heavy metals and trace element concentrations with reproductive hormone levels and polycystic ovary syndrome in a Chinese population. Biol Trace Elem Res. 2015, 167, 1-10. 